\title{
Distribution of HLA antigens in breast cancer
}

\author{
Bayraktar B ${ }^{1}$, Yilmaz E ${ }^{2}$, Bayraktar $\mathrm{O}^{1}$, Apaydin $\mathrm{BB}^{1}$, Erguner $\mathrm{IE}^{3}$, Kayabasi $\mathrm{B}^{1}$, \\ Ozcelik $\mathrm{AA}^{4}$, Eren $\mathrm{B}^{5}$
}

Department of General Surgery, Istanbul University Cerrahpasa Medical School, Istanbul, Turkey. bulenteren2000@yahoo.com

\begin{abstract}
Investigation of various tumor-specific markers has a critical role in early diagnosis and treatment of breast cancer. The aim of the this study is to investigate the Human Leukocyte Antigen (HLA) alleles, the molecules that play an important role in immunity and tumor response of the body, and its relationship with breast cancer. In this prospective clinical study, after obtaining approval from the ethics committee of Istanbul University Experimental Medical Research Institute, 22 female patients who have been hospitalized in Istanbul University Cerrahpasa Faculty of Medicine the Department of General Surgery with a diagnosis of breast cancer were selected. In the control group, there were 22 healthy women who had no relationship and were donor candidates for renal transplantation. After collecting blood in $5 \mathrm{ml}$ tubes with EDTA, HLA A, B and DR groups were measured with SSP method using the GenoVision Olerup SSP (Olerup SSP, Stockholm, Sweden) kit in Istanbul University Cerrahpasa Faculty of Medicine Blood Center Tissue Type Determination Laboratory. In patient and control group, totally 53 alleles; 17 alleles of HLA-A gene, 22 alleles of B gene, 14 alleles of DR gene were detected. A statistically significant relationship was determined between HLA-B55:01 and HLADRb1*18:01 alleles and the development of breast cancer $(p<0.05)$. HLA-B13:01 antigen is determined only in the control group. It was concluded that HLA-B13:01 antigen, determined only in the control group, may be protective for breast cancer and HLA-B55:01 and HLA-DRb1*18:01 antigens, determined only in the patient group, may be a risk factor for breast cancer (Tab. 5, Ref. 22). Full Text in PDF www.elis.sk.

Key words: HLA antigens, breast cancer.
\end{abstract}

Cancer is a serious public health problem in developed countries. According to the World Health Organization's (WHO) data, $12 \%$ of the 56 million deaths are in consequence of identified malignant tumors worldwide in 2000. 5.3 million men and 4.7 million women had malignant tumors and 6.2 million people died as a result of disease (1). Breast cancer is the most common type of cancer in women according to 2002 data. The second most common cause of cancer death in women is breast cancer after cervical cancer in developing countries, and the most common is breast cancer in developed countries (2). Pathogenesis of breast cancer is multifactorial. Breast cancer is thought to develop with genetic predisposition, environmental factors, hormones and even interaction with infectious agents. Today it is known that all species contain a gene part called MHC (Major Histocompatibility Complex), regulating antigen recognition and encoding cell surface glycoprotein by $\mathrm{T}$ lymphocytes (3). According to

${ }^{1}$ Department of General Surgery, Istanbul University Cerrahpasa Medical School, Istanbul, Turkey, ${ }^{2}$ Tissue Typing Laboratory, Istanbul University Cerrahpasa Medical School, Blood Bank,Istanbul, Turkey, ${ }^{3}$ Department of General Surgery, Akademi Hospital, Kocaeli, Turkey ${ }^{4}$ Department of General Surgery, Ministry of Health, Goztepe Training and Research Hospital, Istanbul, Turkey, and ${ }^{5}$ Council of Forensic Medicine of Turkey, Bursa Morgue Department, Bursa, Turkey

Address for correspondence: B. Eren, MD, Council of Forensic Medicine of Turkey, Bursa Morgue Department;16010, Bursa, Turkey.

Phone: +90.224.2220347, Fax: +90.224.2255170 the research HLA (Human Leukocyte Antigen) system, a component of MHC, is not only an important noncompliance factor in transplantation but is also effective in vital biological events such as the immunological recognition, immune response and its relationship with disease. HLA alleles especially play major role in cellular immunity and may be important for the development of breast cancer genetically $(3,4)$. The relationship between HLA and various pathological disorders have been previously reported. The aim of this study is to demonstrate the association of breast cancer with HLA.

\section{Materials and methods}

After obtaining approval from Istanbul University Institute of Experimental Medical Research Ethics Committee, 22 female patients with breast cancer diagnosis and average age of 48.2, hospitalized at Istanbul University Cerrahpasa Medical Faculty, Department of General Surgery for operations, were selected as the patient group. As the control group 22 healthy unrelated women, with assigned tissue groups because of being donor candidates for renal transplantation and a mean age of 45 were selected. Blood samples were collected in $5 \mathrm{ml}$ tubes with EDTA, and were analyzed at Istanbul University Cerrahpasa Medical Faculty Blood Center Tissue Typing Laboratory. HLA typing was performed using GenoVision Olerup SSP kit (Olerup SSP, Stockholm, Sweden). HLA A, B and DR groups were measured. 
Tab. 1. Detected HLA- A alleles.

\begin{tabular}{|c|c|c|c|c|c|c|c|}
\hline & \multicolumn{2}{|c|}{ Study } & \multicolumn{2}{|c|}{ Control } & \multirow{2}{*}{$\mathrm{p}$} & \multirow{2}{*}{ OR } & \multirow{2}{*}{ CI $(\% 95)$} \\
\hline & $\mathrm{n}$ & $\%$ & $\mathrm{~N}$ & $\%$ & & & \\
\hline A01:01 & 6 & 27,3 & 3 & 13,6 & 0,262 & 2,37 & $0,51-11,04$ \\
\hline A02:01 & 9 & 40 & 9 & 40 & 0,1 & 0,3 & $0,3-3,32$ \\
\hline A03:01 & 3 & 13,6 & 6 & 27,3 & 0,262 & 0,42 & $0,09-1,95$ \\
\hline A11:01 & 5 & 22,7 & 1 & 4,5 & 0,79 & 6,17 & $0,65-58,03$ \\
\hline A19:01 & 3 & 13,6 & 0 & - & 0,073 & - & $0,98-1,36$ \\
\hline A23:01 & 2 & 9,1 & 0 & - & 0,148 & - & $0,96-1,255$ \\
\hline A24:01 & 6 & 27,3 & 6 & 27,3 & 1 & 1 & $0,26-3,76$ \\
\hline A26:01 & 2 & 9,1 & 3 & 13,6 & 0,635 & 0,63 & $0,09-4,21$ \\
\hline A28:01 & 2 & 9,1 & 0 & - & 0,148 & - & $0,96-1,25$ \\
\hline A29:01 & 1 & 4,5 & 1 & 4,5 & 1 & 1 & $0,06-17,06$ \\
\hline A30:01 & 1 & 4,5 & 2 & 9,1 & 0,55 & 0,47 & $0,40-5,67$ \\
\hline A32:01 & 3 & 13,6 & 3 & 13,6 & 1 & 1 & $0,17-5,59$ \\
\hline A33:01 & 0 & - & 2 & 9,1 & 0,148 & - & $0,79-1,03$ \\
\hline A68:01 & 1 & 4,5 & 2 & 9,1 & 0,55 & 0,476 & $0,4-5,67$ \\
\hline
\end{tabular}

Tab. 2. Detected HLA-B alleles.

\begin{tabular}{|c|c|c|c|c|c|c|c|}
\hline & \multicolumn{2}{|c|}{ Study } & \multicolumn{2}{|c|}{ Control } & \multirow{2}{*}{$\mathrm{p}$} & \multirow{2}{*}{ OR } & \multirow{2}{*}{$\mathrm{CI}(\% 95)$} \\
\hline & $\mathrm{n}$ & $\%$ & $\mathrm{~N}$ & $\%$ & & & \\
\hline B04:01 & 1 & 4,5 & 1 & 4,5 & 1 & 1 & $0,06-17,06$ \\
\hline B05:01 & 0 & - & 1 & 4,5 & 0,312 & - & $0,87-1,04$ \\
\hline B07:01 & 2 & 9,1 & 2 & 9,1 & 1 & 1 & $0,12-7,81$ \\
\hline B08:01 & 3 & 13,6 & 3 & 13,6 & 1 & 1 & $0,17-5,59$ \\
\hline B13:01 & 0 & - & 4 & 18,2 & 0,036 & - & $0,67-0,99$ \\
\hline B14:01 & 0 & - & 2 & 9,1 & 0,148 & 0,909 & $0,79-1,03$ \\
\hline B15:01 & 0 & - & 2 & 9,1 & 0,148 & - & $0,79-1,03$ \\
\hline B17:01 & 1 & 4,5 & 0 & - & 0,312 & - & $0,95-1,14$ \\
\hline B18:01 & 1 & 4,5 & 4 & 18,2 & 0,154 & 0,214 & $0,02-2,09$ \\
\hline B21:01 & 1 & 4,5 & 0 & - & 0,312 & - & $0,95-1,14$ \\
\hline B55:01 & 4 & 18,2 & 0 & - & 0,036 & - & $1-1,48$ \\
\hline B27:01 & 4 & 18,2 & 1 & 4,5 & 0,154 & 4,667 & $0,47-45,6$ \\
\hline B38:01 & 2 & 9,1 & 2 & 9,1 & 1 & 1 & $0,12-7,81$ \\
\hline B44:01 & 6 & 27,3 & 5 & 18,2 & 0,472 & 1,688 & $0,4-7,07$ \\
\hline B51:01 & 4 & 18,2 & 3 & 13,6 & 0,68 & 1,407 & $0,27-7,18$ \\
\hline B52:01 & 0 & - & 2 & 9,1 & 0,148 & - & $0,79-1,03$ \\
\hline B53:01 & 0 & - & 3 & 13,6 & 0,073 & - & $0,73-1,02$ \\
\hline
\end{tabular}

Tissue typing with PCR-SSP methods

$3 \mathrm{ml}$ of blood samples were taken from patients and donors sent from other clinics for HLA-A, B and DR typing, and were put into tubes with EDTA. DNA isolation from the stored blood samples was made with standard protocols (5). DNA purity was calculated spectrophotometrically at 260/280 nm wave length, and its concentration was regulated. Twenty-three different primary for HLA-A, 48 for HLA-B, 23 for HLA-DR were used. PCR materials may be received and prepared separately or may be provided as partially ready on commercial basis.

After PCR reaction was installed, it was placed in the thermal cycle device. PCR reaction consists of 3 different phases:

1) DNA denaturation,

2) Matching the primaries,

3) Replication with the Taq polymerase.

This third phase consisted of different temperatures and is counted as 1 cycle. Reaction was terminated after 25-30 cycles. The obtained PCR products were forwarded in a $2 \%$ agarose gel electrophoresis (1XTBE or 0.5 XTBE). Then tissue groups were determined by photographing in UV transilluminator. Results were written by grouping according to the allelic or serological numbering system. Internal controls were always checked for proper working (6). Olerup et al have determined that genomic HLA-A, $\mathrm{B}, \mathrm{C}, \mathrm{DR}$ typing is much more accurate and sensitive (99.2\%) than serological methods (6-8).

\section{Statistical analysis}

Statistical analysis was performed by using SPSS on Windows computer program. HLA's of patient and control group were compared using Chi square test and Odd's Ratio (OR) that were calculated for HLA's, having difference according to the $\mathrm{p}<0.05$ significance level.

\section{Results}

In the present study, patient group consisted of 22 women with breast cancer; control group consisted of 22 healthy women. The age of women in patient group ranged between 28 and 79 years and the mean age was 48.2. The age of women in study group 
Tab. 3. Detected HLA- DR alleles.

\begin{tabular}{|c|c|c|c|c|c|c|c|}
\hline & \multicolumn{2}{|l|}{ Study } & \multicolumn{2}{|c|}{ Control } & \multirow{2}{*}{$\mathrm{p}$} & \multirow{2}{*}{ OR } & \multirow{2}{*}{$\mathrm{CI}(\% 95)$} \\
\hline & $\mathrm{n}$ & $\%$ & $\mathrm{~N}$ & $\%$ & & & \\
\hline DRB1*0101 & 1 & 4,5 & 3 & 13,6 & 0,294 & 0,302 & $0,03-3,15$ \\
\hline DRB1*02:01 & 1 & 4,5 & 0 & - & 0,312 & - & $0,95-1,14$ \\
\hline DRB1*03:01 & 1 & 4,5 & 4 & 18,2 & 0,154 & 0,214 & $0,02-2,09$ \\
\hline DRB $1 * 04: 01$ & 6 & 27,3 & 7 & 31,8 & 0,741 & 0,804 & $0,21-2,94$ \\
\hline DRB $1 * 0701$ & 2 & 9,1 & 3 & 13,6 & 0,635 & 0,633 & $0,09-4,21$ \\
\hline DRB $1 * 08: 01$ & 2 & 9,1 & 1 & 4,5 & 0,550 & 2,1 & $0,17-25$ \\
\hline DRB1*13:01 & 4 & 18,2 & 6 & 27,3 & 0,472 & 0,593 & $0,14-2,48$ \\
\hline DRB1*15:01 & 6 & 27,3 & 3 & 4,5 & 0,262 & 2,375 & $0,51-11$ \\
\hline DRB $1 * 16: 01$ & 1 & 4,5 & 5 & 22,7 & 0,079 & 0,162 & $0,01-1,52$ \\
\hline DRB1*18:01 & 5 & 22,7 & 0 & - & 0,018 & - & $1,03-1,62$ \\
\hline
\end{tabular}

Tab. 4. HLA antigens, statistically significant.

\begin{tabular}{lccc}
\hline & Breast Cancer & Control & $\mathrm{p}<0,05$ \\
\hline HLA- B13:01 & 0 & 4 & 0,036 \\
HLA- B55:01 & 4 & 0 & 0,036 \\
HLA- DRB1 $18: 01$ & 5 & 0 & 0,018 \\
\hline
\end{tabular}

ranged between 23 and 60 years and the mean age was 45 years. Pathologies of all patients in the study group were invasive ductal breast cancer. Women in the control group were healthy donor candidates for renal transplantation. All the sub-groups of HLAA, B, DR genes were examined in Blood Center Tissue Type Determination Laboratory in Istanbul University Cerrahpasa Medical School (Tabs 1, 2 and 3). In the study group; 14 alleles of HLA-A gene, 15 alleles of HLA-B gene, 13 alleles of HLA-DR gene were determined. In the control group; 12 alleles of HLA-A gene, 16 alleles, of HLA-B gene, 12 alleles of HLA-DR gene were determined. In both groups; 17 alleles of A gene, 22 alleles of B gene and 14 alleles of DR gene, total of 53 alleles, were detected. HLA-B13:01, was not detected in the patient group, but was detected in 4 women in the control group. The difference was statistically significant $(p<0.05)$. Reliance interval was determined as $0.67-0.99$. This antigen was detected only in the control group and was thought to have a protective activity. In patient group, HLA-B55:01 was detected in 4 women and HLA-DRB1*18:01 was also in 4 women. Both antigens were detected in no subject in the control group. Reliance interval of antigens, determined statistically significant ( $\mathrm{p}<0.05)$, was 1-1.48 for HLA-B55:01 and 1.03-1.62 for HLADRB1*18:01 (Tab. 4).

\section{Discussion}

Breast cancer has a tendency of familial accumulation, suggesting that genetic factors might have a role in the etiology (9). There are many studies emphasizing the relationship between HLA system and some diseases. The aim of the present study was to establish the relationship between HLA antigens and breast cancer (10-13). Several studies (Tab. 5), investigating the relationship between breast cancer and HLA were examined since 1970s (10-14). Patel et al and Iaffaioli et al in their studies determined that there was a significant relationship between HLA B7 and breast cancer and the common characteristics of those who carry these antigens were premenopausal status, the lack of hormone receptors and primary tumors with high histological grade (14-15). In studies of Boulinelle et al, a statistically significant relationship was defined between breast cancer and HLA-A28 and identified that this antigen was common in postmenopausal, especially nulliparous, women (16). Emekcioglu et al determined that HLA-B7, B17, Bw50 and DR4 were associated with breast cancer (4). Casole et al detected a significant relationship between breast cancer and HLA-B7 and DR4 (17), Lavado detected this relationship only with HLA-B7 (18). HLA-DRB $1 * 1101, \mathrm{DQB} 1 * 03032$ alleles were determined to be protective against breast cancer by Chaudhuri et al (10). Ghaderi et al defined that the frequency of 12. allele on HLA DRB1 was significantly higher (11). Harrath et al established a reverse link among HLA-DRB1*07 and HLA-DRB1*02 alleles and breast cancer(12).

MHC expression of the tumor has been thought to be effective in response to treatment during, before or after immunotherapy. In

Tab. 5. HLA antigens, associated with breast cancer.

\begin{tabular}{lll}
\hline HLA antigen & Authors & Association with HLA \\
\hline HLA B7 & Patel (14) and İaffaioli (15) & Determined significant association \\
HLA A28 & Boulinelle et al. (16) & Determined significant association. Especially with postmenapause and nullipars \\
HLA B7, B17, Bw50 and DR4 & Emekcioglu et al (4) & Determined association with breast cancer \\
HLA B7, HLA DR4 & Casoli C. Et al. (17) & Determined significant correlation \\
HLA B7 & Lavado et al. (18) & Determined significant correlation \\
HLA DRB1, DQB1 & Chaudhuri S. et al. (10) & DRB1*1101, DQB1*03032 allelles were determined to be protective for breast \\
HLA DRB1 & Ghaderi A. et al. (11) & 12. allele frequency was detected significantly higher in HLA DR B1 \\
HLA DRB1 & Harrath AB. et al. (12) & HLA DRB1*07 and HLA DRB1*02 allelles were determined to have a reverse link with \\
& & breast cancer \\
HLA class I & Madjd Z. et al. (13) & Total loss of MHC class I was defined to be an independent marker for good prognosis \\
& & in breast cancer \\
\hline
\end{tabular}


some studies HLA Class I expression was examined in premalignant lesions or in tissues with high-risk for tumor formation. Thus, in the familial and sporadic colonic carcinogenesis, HLA antigens were found to decrease in the normal mucosa close to adenoma as well as in adenomas (19). Madjd et al determined in their study in women with breast cancer that total loss of MHC class I was an independent marker for good prognosis in breast cancer (13). Effect of hormones might have a role on the expression of HLA antigens and effects the regulation of the behavior of tumor indirectly. In fact, HLA Class I expression is induced in MCF-7 cell lines after the addition of estrogen into the place $(20,21)$. However Redondo et al. could not define a significant relationship between estrogen receptors and HLA Class I antigens (22). In the present study HLA typing is planned in patients with breast cancer and in healthy donor candidates for renal transplantation. As a result, HLA-B55:01 and HLA-DRB1*18:01 were determined significantly higher in the patient group. It was thought that both HLA antigens cause predisposition for breast cancer, and HLA-B13:01 antigen, detected significantly higher in control group, provides protective activity. The results of the present study do not comprise a common antigen with other studies in the literature, but the reason seems to be because of the low number of patients and lack of studies. When the results were evaluated, HLA DR18 and HLA-B55:01 positive people, having other risk factors, are at risk for breast cancer and they should be considered for examination and follow-up should be planned.

\section{Conclusion}

Early diagnosis and treatment of breast cancer is evolving rapidly with advancing technology and progress in the science of genetics. However, breast cancer is still the most common cause of cancer death of women in developed countries. So HLA allele frequencies of different populations, at risk for breast cancer, and various sub-groups of HLA should be investigated for assessing the important alleles, specified as risk factors of development of breast cancer.

\section{References}

1. World Health Organisation. World Health Report 2001. Mental Health: New Understanding, 2001. New Hope. Geneva: WHO.

2. Parkin DM, Bray F, Ferlay J, Pisani P. Global cancer statistics. 2002. CA Cancer J Clin 2005; 55 (2): 74-108.

3. Chen PC, Tsai EM, Er TK, Chang SJ, Chen BH.HLA-DQA1 and -DQB1 allele typing in southern Taiwanese women with breast cancer. Clin Chem Lab Med 2007; 45 (5): 611-614.

4. Emekcioglu S, Altun M, Sönmez G, Hamizade N.M, Carin N. HLA distribution in breast cancer. Cancer 1989; 4 (1-3): 774-777.

5. Miller SA, Dykes DD, Polesky HF. A simple salting out procedure of extracting DNA from human nucleated cells. Nucleic Acids Res 1988 16: 1215
6. Olerup $\mathbf{O}$, Zetterquist $\mathbf{H}$. HLA-DR typing by PCR amplification with sequence-spesific primers (PCR-SSP) in 2 hours: an alternative to serological DR typing in clinical practice including donor-recipient matching in cadaveric transplantation. Tissue Antigenes 1992; 39 (5): 225-235.

7. Carin M. Transplantation Immmunology(HLA system). J Clin Develop 1997; 10: 7-11.

8. Gurtekin M, Aydın F, Oguz FS. Selection of Donor for bone marrow transplant patients, the compliance of mixed lymphocyte culture test and genotyping. J Clin Develop 1997;10: 12-16.

9. Baykal A, Sahin AA. Pathology and molecular biology of breast disease. Ed. I. Sayek. Basic Surgery, 3rd Edition. Güneş Kitabevi Medicine Ltd, Ankara, 2004; 929-942.

10. Chaudhuri S, Cariappa A, Tang M, Bell D, Haber DA, Isselbacher KJ. Genetic susceptibility to breast cancer: HLA DQB1 *03032 and HLA DRB1*11 may represent protective alleles. PNAS 2000; 97: 11451-11454.

11. Ghaderi A, Talei A, Gharesi-Fard B, Farjadian SH, Amirzargar A, Vasei. HLA-DBR 1 alleles and the susceptibility of Iranian patients with breast cancer. Pathol Oncol Res 2001; 7 (1): 39-41.

12. Harrath AC, Loueslati BY, Troudi W, Hmida S, Sedkaoui S, Ayed FB, Rhomdhane KB, Elgaaied ABA. HLA class II polymorphism: Protective or risk factors to breast cancer in Tunisia. Pathol Oncol Res 2006; 2: 79-81.

13. Madjd Z, Spendlove I, Pinder SE, Ellis IO, Durrant LG. Total loss of MHC class I is an independent indicator of good prognosis in breast cancer. Int J Cancer 2005; 117 (2): 248-255.

14. Patel R, Habal MB, Wilson RE, Birtch AG, Moore HD. Histocompatibility antigens and cancer of the breast. Am J Surg 1972; 124: 34-43.

15. Iaffaioli RV, Maio M, Ruggiero G, De Felice M, Ungora A, Del Vecchio L, Rosato GB, Bianco AR, Zappacosta S. HLA and prognostik factors in primary breast carcinoma. Int J Cancer 1985; 35: 581-585.

16. Bouillenne C, Deneufbourg JM. Positif correlation between breast cancer incidence and HLA antigens. Oncology 1979; 36: 156-159.

17. Casoli C, Zanelli P, Adorni A, Starcich BR, Neri T. Serological and molecular study on the HLA phenotype of female breast cancer patients. Eur J Cancer 1994; 30A (8): 1207-1208.

18. Lavado R, Benavides M, Villar E, Ales I, Alonso A, Caballero A. The HLA-B7 allele confers susceptibility to breast cancer in Spanish women. Immunol Lett 2005; 101 (2): 223-225.

19. Rolland P, Deen S, Scott I, Durrant L, Spendlove I.Human leukocyte antigen class I antigen expression is an independent prognostic factor in ovarian cancer. Clin Cancer Res 2007; 13 (12): 3591-3596.

20. Rodriguez F, Peran F, Garrido F, et al. Up-modulation by estrogen of HLA class I expression in breast tumor cell lines. Immunogenetics 1994; 39; 161-167.

21. Teh M, Hui KM. Modulation of MHC gene exspression in human breast carcinoma cells by hormones. J Immunogenet 1989; 16: 397-405.

22. Redondo M, Garcia J, Villar E, Rodrigo I, Perea-Milla E, Serrano A, Morell M. Major histocompatibility complex status in breast carcinogenesis and relationship to apoptosis. Hum Pathol 2003; 34 (12): 1283-1289. 